\title{
A aplicabilidade da humanização no atendimento aos usuários do sistema único de saúde
}

\author{
The applicability of humanization in service to users of the national unified health system \\ La aplicabilidad de la humanización em el servicio a los usuarios del sistema único de \\ salud
}

Áchelles Monise Batista da Silva ${ }^{1 *}$, Millena de Carvalho Pereira ${ }^{1}$, Isabelle Lira Amorim Xavier ${ }^{1}$, Anna Flávia Montenegro Lisboa ${ }^{1}$, Yure Suassuna Cardoso', Thais Rodrigues Alcântara ${ }^{1}$, Klenia Felix de Oliveira Bezerra'.

\section{RESUMO}

Objetivo: Analisar as publicações científicas, no período de 2015 a 2020, acerca da aplicabilidade da humanização no atendimento aos usuários do Sistema Único de Saúde dentro de uma relação médicopaciente de qualidade. Resultados: Entre um total de 25 fontes consultadas, destaca-se a relevância da criação do Sistema Único de Saúde e da inserção e aplicabilidade da humanização do cuidado no atendimento aos usuários desse sistema, a partir da implementação e da aplicação dos seus princípios e diretrizes nos serviços de saúde do Sistema Único de Saúde. Considerações Finais: A partir do que foi observado, firma-se a importância da aplicação dos princípios e diretrizes da política nacional de humanização no sistema público de saúde para que, mesmo com as suas fragilidades identificadas, possam ser definidas iniciativas que busquem aplicar uma comunicação médico-paciente-gestão de maior qualidade nos serviços e, consequentemente, seja possível proporcionar uma maior eficiência na promoção e na prevenção da saúde desses usuários.

Palavras-chave: Medicina integrativa, Sistema único de saúde, Saúde pública, Humanização.

\begin{abstract}
Objective: To discuss the applicability of humanization in the care of users of the Unified Health System within the doctor-patient relationship. Methods: The study is a basic, descriptive, secondary source, qualitative, historical, and bibliographic review typically carried out at the Virtual Health Library (VHL) and intensified by the experience of the authors in the field of activity of public health. Results: It is observed the relevance of the creation of the Unified Health System and the insertion of humanization in the service to the user in this system, from the implementation and application of principles and guidelines in health services, since there is greater efficiency in the promotion and health prevention based on a good doctor-patient relationship. Conclusion: Based on what was observed, it's confirmed the importance of applying the principles and guidelines of the national humanization policy in the public health system is confirmed so that, even with its identified weaknesses, it can define initiatives that seek to apply a high-quality doctor-patient communication in health services and, consequently, provide greater efficiency in promoting and preventing the health of these users.
\end{abstract}

Keywords: Integrative medicine, Unified health system, Public health, Humanization.

${ }^{1}$ Faculdade Ciências Médicas da Paraíba (FCM/PB), Cabedelo - PB. *E-mail: achellesmonise@gmail.com 


\section{RESUMEN}

Objetivo: Analizar publicaciones científicas, de 2015 a 2020, sobre la aplicabilidad de la humanización en la atención a los usuarios del Sistema Unificado de Salud dentro de la relación médico-paciente. Métodos: Se trata de un estudio descriptivo, con un enfoque cualitativo, de la revisión narrativa de la literatura. Las fuentes fueron seleccionadas de las bases de datos de la Biblioteca Virtual de Salud (VHL), así como documentos administrativos de organismos gubernamentales brasileños. Revisión Bibliográfica: De las 25 fuentes consultadas, destaca la relevancia de la creación del Sistema Unificado de Salud y la inserción de la humanización en la atención del usuario en este sistema, de la implantación y aplicación de principios y directrices en los servicios de salud. Conclusión: Se establece la importancia de la política nacional de humanización en el sistema de salud pública, que, incluso con las debilidades identificadas, define directrices, que buscaban mejorar la comunicación médico-paciente-gestión en los servicios y, en consecuencia, proporcionar una mayor eficiencia en la promoción y prevención de la salud.

Palabras clave: Medicina integrativa, Sistema unificado de salud, Salud pública, Humanización.

\section{INTRODUÇÃO}

A história das políticas públicas no Brasil teve grandes avanços com o passar dos anos e um dos principais, foi a criação do Sistema Único de Saúde (SUS). O SUS foi criado pela necessidade da garantia do direito à saúde, oferecido na Constituição Federal de 1988. Antes de sua criação, as ações de promoção e os programas de saúde eram escassos. Com esse novo sistema, pretendia-se a garantia da assistência integral à saúde, de forma completamente gratuita, a toda a população brasileira e até para estrangeiros, que estivessem no país e precisassem de algum acompanhamento de saúde (BRASIL, 2017c; BRASIL, 2019).

No entanto, ao longo do tempo, começaram a aparecer problemas na qualidade das práticas de saúde do SUS, como a superlotação do sistema, uma vez que os princípios de acolhimento eram ainda escassos no sistema e o que se buscava eram tentativas de atender à alta demanda. Nesse sentido, com o objetivo de melhorar os serviços de saúde e garantir a comunicação, favorecendo atitudes solidárias entre gestores, trabalhadores e usuários do sistema, foi criada, em 2003, a Política Nacional de Humanização (PNH) (BRASIL, 2017b).

Todavia, mesmo com a criação desse projeto, ainda é possível observar problemas relacionados à falta de humanização no atendimento ao público no Sistema Único de Saúde, devido à existência de desafios como pouco acesso a programas de capacitação pelos profissionais que compõem o sistema, bem como problemas na sua gestão (PEREIRA LB e CHAZAN AC, 2019).

Assim, mesmo após a criação de mecanismos que ajudem a integrar não só a relação médico-paciente, bem como de todos os que compõem o Sistema Único de Saúde, percebe-se, ainda, deficiência na oferta da humanização a esse público.

Nesse sentido, o estudo levantou o seguinte questionamento: Por quê, mesmo depois da criação da Política de Humanização, ainda ocorrem desafios na relação médico-paciente, bem como na implementação de métodos mais eficientes para efetivar esta habilidade? Neste contexto, o estudo teve como objetivo analisar a aplicabilidade da humanização no atendimento aos usuários do Sistema Único de Saúde dentro da relação médico-paciente.

\section{REVISÃO BIBLIOGRÁFICA}

O Sistema Único de Saúde (SUS) é um dos sistemas de saúde pública mais complexos do mundo que tem o objetivo de atuar na promoção, prevenção e recuperação da saúde, que promove acesso de atenção integral à saúde. Nesse viés, para que haja uma melhor compreensão desse sistema, torna-se necessário entender a sua relação com a história político-social da sociedade brasileira (BRASIL, 2019). 
O SUS foi criado no contexto de redemocratização da política brasileira, em que se buscava a garantia de direitos igualitários para toda a população. No âmbito das políticas públicas de saúde, anteriores à criação do SUS houve muitos movimentos populares e a criação de projetos que abrangesse melhores condições de saúde à população. Isso porque é possível observar que a situação de saúde da população tinha um perfil epidemiológico com predomínio, principalmente, de doenças infecciosas transmissíveis, uma vez que, com o crescimento das cidades, havia muitos aglomerados populares e o saneamento básico do país era precário, fatos que contribuem para o cenário de proliferação dessas doenças (BRASIL, 2017b).

Ainda, o acesso à saúde era inicialmente restrito a pessoas da alta sociedade, e apenas após avanços de vários movimentos trabalhistas, com a criação de institutos que garantiam a assistência popular, foi estendido o atendimento aos trabalhadores previdenciários, que tinham um acesso mais amplo à saúde, e, posteriormente, aos não previdenciários, que tinham acesso mais limitado (BRASIL, 2017b). Dessa forma, percebe-se que as condições sociais de saúde eram constituídas por muitas carências e barreiras de atenção à população, mesmo após a criação de projetos que buscavam garantir maior acesso à saúde. Logo, é possível perceber a necessidade da criação de políticas públicas que verdadeiramente garantissem o acesso de toda a população a uma saúde de qualidade (BRASIL, 2017b).

Assim, apenas com a Constituição Federal de 1988, foram criadas leis que garantiam a saúde como dever do Estado e direito da população, com acesso livre, universal e igualitário. Para suprir tal direito, foi criado o Sistema Único de Saúde, organizado pelo Ministério da Saúde, o qual deveria formular, fiscalizar e monitorar as políticas e ações do sistema. Além disso, o SUS é baseado na descentralização de forma não hierarquizada, em que há a contribuição de gestão da União, que é o principal financiador da saúde pública do país. Além da União, o SUS é também uma responsabilidade dos Estados, os quais são responsáveis pelas políticas de saúde do território, e Municípios, os quais devem oferecer os serviços de saúde da população. Dessa forma, torna-se possível garantir o seu funcionamento de acordo com a determinação constitucional (BRASIL, 2019).

O SUS oferece acesso integral e universalizado sem custo a todos os brasileiros e estrangeiros que estejam no país em todos os níveis de saúde. Assim, para garantir a qualidade e organização dos serviços, o sistema divide suas atividades em redes de atenção à saúde que operam de forma cooperativa e com intercâmbio de objetivos e de recursos, em uma organização sem hierarquia, que oferecem a atenção de forma integrada e organizada de forma integral e multidisciplinar, que abrange todos os níveis de atenção, que são divididos e distribuídos de acordo com a necessidade da população (BRASIL, 2019).

Os níveis são divididos em quatro: primário, que atende à atenção básica e que é o ponto de contato referencial dos usuários do SUS, além de apresentar ações de promoção da saúde como prevenção de doenças, redução de danos e reabilitação; o nível secundário, que visa atender o usuário do sistema com uma prática que demande de profissionais mais especializados que os do nível primário; o nível terciário, que é composto principalmente pela atenção hospitalar, com procedimentos que envolvem alta densidade tecnológica como cirurgias; e o nível quaternário, que envolve ações mais invasivas, como transplantes (BRASIL, 2017a; SOUSA IM e TESSER CD, 2017; GROSS DM, 2016). Assim, é possível perceber a importância dessa política pública, uma vez que seu papel é universalizar e eliminar barreiras geográficas, econômicas e culturais entre a população (PEREIRA LB e CHAZAN AC, 2019; MATTOS G, et al., 2018).

Além dos níveis, o SUS também conta com princípios e diretrizes que ajudam na sua organização. Os princípios, que são ideias estruturantes do sistema, enquanto as diretrizes, que são meios por onde pretendem-se atingir o objetivo de existir e que conferem legitimidade a si (BRASIL, 2019).

Sobre os princípios, eles se dividem em três, os quais são: Universalidade, Equidade e Integralidade. A Universalidade, é o princípio que tem a saúde como um direito de todos e dever do Estado e o acesso à bens e serviços deve ser garantido a todos os usuários, independe de sexo, cor, raça, religião e demais características sociais e/ou pessoais. A Equidade, tem o objetivo de diminuir as desigualdades, ou seja, tratar desigualmente os desiguais, investindo mais onde existe mais carência. Por fim, a Integralidade, que considera o ser humano em sua totalidade e visa atender todas as suas necessidades, integrando todos os níveis de atenção e que usa da articulação da saúde com outras políticas públicas, para assegurar o bemestar e a qualidade de vida dos usuários (MATTA GC, 2007). Já em relação as diretrizes, destaca-se a Descentralização, a Regionalização e hierarquização e a Participação da comunidade (MATTA GC, 2007). 
A Descentralização, corresponde à distribuição de responsabilidades e de recursos da esfera federal para estadual e municipal, com o intuito de retirar a concentração total de poder da União e distribuí-lo para Estados e Municípios, dando a eles a autonomia necessária. A Regionalização e hierarquização busca aproximar os gestores dos problemas e condições de saúde do município e suas regiões, fazendo com que quanto mais próximo dos usuários, o sistema tenha maior capacidade de identificar os problemas e necessidades de saúde e agir mais objetivamente na promoção do acesso a bens e serviços de saúde para a população. Assim, percebe-se que a Regionalização é norteada pelos níveis de complexidade, que vão desde a promoção e prevenção até a intervenções de maior complexidade, como transplantes. Ainda, a diretriz da Participação da Comunidade visa dar voz aos usuários, buscando ouvir as necessidades da população através de instâncias colegiadas como os conselhos e as conferências de saúde (MATTA GC, 2007).

Nesse viés, embora muito bem estruturado em sua constituição e legislação, o Sistema Único de Saúde enfrenta diversas dificuldades na prática. Estudos apontam que os maiores desafios para uma boa funcionalidade do Sistema de Saúde são a falta de profissionais qualificados, a demora para atendimentos em todos os níveis de saúde, a demora para conseguir consultas, a falta de medicamentos nas unidades básica de saúde (UBS), exame físico e anamnese insatisfatórios, além das enormes filas que os usuários enfrentam diariamente (JASMIM JS e QUELUCI G, 2018).

Assim, percebe-se que os profissionais, que trabalham neste ambiente, por muitas vezes exauridos de trabalho, também em virtude da deficiência do número de profissionais nas regiões, acabam não atendendo seus usuários de forma correta e humanizada, prezando pela boa comunicação e bom entendimento. Porém, é de extrema relevância ressaltar que a boa comunicação e o olhar holístico sobre o usuário são imprescindíveis para um bom atendimento e uma boa comunicação (ARAUJO AK, et al., 2015).

Neste contexto, surge a necessidade de mudança de práticas, de um atendimento mais humanizado. Então, em 1978, ocorreu a declaração de Alma-Ata, que tinha como alvo a criação de uma rede de saúde que contemplasse o bem-estar físico, mental e social, promovendo educação em saúde para maior prevenção, proteção, imunização e controle da saúde, além de acesso a nutrição adequada, implementação de saneamento básico e planejamento familiar. Essa declaração propunha que as instituições de saúde fossem centradas nas necessidades da população em uma escala interdisciplinar, que envolvesse os profissionais da saúde, incluindo também a participação da população para o controle de suas atividades (FERNANDES VC, et al., 2017). A Alma-Ata serviu como base para a Constituição Federal e para a implementação do Sistema Único de Saúde (SUS) (FERNANDES VC, et al., 2017). Mas, só em 2003, que foi criada a Política Nacional de Humanização (PNH), com o objetivo de efetivar os princípios do SUS em todos os seus programas (BRASIL, 2017c)

A humanização consiste em ver o paciente de forma integral (corpo, mente e alma), não focar somente na doença, como no antigo modelo. Ela leva em consideração o emocional do indivíduo, a capacidade de lidar com as diferenças, sejam elas financeiras, religiosas ou étnicas. Ainda, existe 0 ato da sensibilidade, percepção das necessidades alheias, escuta do paciente, a fim de compreendê-lo melhor, de ver o processo saúde-doença de modo ampliado, que visa o cuidado e tem foco na prevenção, além de contribuir com uma maior autonomia ao paciente e capacidade de transformar a realidade em que vivem (ASSIS WC, et al., 2018; SANDES LF, et al., 2018; MATOS PC, et al., 2018).

A Política Nacional de Humanização (PNH) do SUS visa a comunicação entre gestores, profissionais e usuários, a fim de gerar planos coletivos para existir uma boa relação de afeto e poder, que na maioria das vezes produzem atitudes desumanizadas e impedem a boa relação entre os trabalhadores da área de saúde. 1 No entanto, mesmo com essa implementação, pesquisas ainda apontam que existem alguns desafios em inserir essa prática atualmente, uma vez que muitos profissionais optam por um atendimento rápido, sem interação, ainda visando só no lucro e, isso acontece pelo antigo modelo ainda enraizado na sociedade. Apesar disso, esse índice tem diminuído cada vez mais, devido às práticas que foram inseridas com o passar dos anos, como as propostas pela $\mathrm{PNH}$, e também, pelo novo método de ensino da área da saúde, que influencia para um atendimento mais humano, visto que os estudantes dessa área são inseridos no campo de prática prematuramente desde os primeiros passos no curso e isso permite uma maior e mais eficaz aproximação com a realidade (SANTOS TD, et al., 2018) 
Uma das ferramentas inclusas que colaboraram com o aumento de espaços de saúde mais humanizados na saúde, foi a implementação das práticas integrativas e complementares à saúde (PICS), também conhecidas como medicina tradicional e medicina complementar ou alternativa, foi uma das ferramentas inclusas que colaboraram com o aumento de espaços de saúde mais humanizados (CARVALHO JL e NOBREGA MP, 2017; CONTATORE AO, et al., 2015; SOARES DP, et al., 2019).

As PICS constituem-se de condutas que promovem a saúde, tanto física quanto mental, a exemplo da meditação, yoga, aromaterapia, terapia comunitária familiar, homeopatia, acupuntura, musicoterapia, biodança e fitoterapia. Essas práticas promovem redução do estresse, alívio da sensação dolorosa e do cansaço físico e psíquico além da manutenção da saúde mental. Ainda, fora esses inúmeros benefícios, há uma redução de gastos, pois a sua inserção é de valor reduzido, unificando eficácia terapêutica com viabilidade financeira, tornando esta implementação viável as redes de saúde pública (ASSIS WC, et al., 2018; MATOS PC, et al., 2018). Com isso, fica evidente que a introdução dessas práticas, juntas à humanização, tornam os atendimentos mais prestativos, com tratamentos paliativos, procedimentos não invasivos e atendendo e respeitando sempre a vontade e a autonomia do paciente (CONTATORE AO, et al., 2015).

A atuação da área da medicina deve ser respaldada na inclusão, além de promover o respeito e à cooperação, o qual deve ter uma educação baseada na necessidade de saúde da população, respeitando as diversidades (PEREIRA LB e CHAZAN AC, 2019; BLUM RA et al., 2018). Assim, diante das fragilidades na assistência dos médicos em muitos lugares, foi criado o Programa Mais Médicos (PMM), cuja função era melhorar as relações médico-pacientes e contribuir na melhoria do atendimento e assistência em lugares com carência destes profissionais. Estudos recentes apontam que o médico o atendimento depois da inserção do PMM melhorou em $87,3 \%$. Essa melhora deve-se a assiduidade do profissional na unidade de saúde e a sua disponibilidade (MOURÃO NETTO JJ, et al., 2018). Houve também uma contribuição significativa na criação das Práticas Integrativas e Complementares (PIC), na medida que permitiu uma visão mais humanizada, de forma a visualizar o paciente como um todo, a tríade corpo-mente-alma (ASSIS WC, et al., 2018; CARVALHO JL e NOBREGA MP, 2017; CONTATORE AO, et al., 2015).

No contexto da saúde indígena, um estudo nacional envolvendo 613 indígenas relatou que $47 \%$ afirmaram que a equipe de saúde que atende a comunidade não incluía médico antes do início do PMM. Isso mostra que, mesmo em caráter emergencial, essa necessidade foi suprida, uma vez que, em 2015, o programa estava em todos os Distritos Sanitários Especiais Indígenas (DSEI) do país, fazendo com que, pela primeira vez, todos contassem com médicos. Sendo assim, além da população que vive nos centros urbanos, os indígenas também foram favorecidos com a implementação do programa mais médicos (PMM). De acordo com um estudo, realizado com 618 indígenas, descobriu-se que antes da implementação do PMM, não havia inserção de médicos pela equipe de saúde para atendê-los. Portanto, em 2015, todos os Distritos Sanitários Especiais Indígenas (DSEI) apresentavam a participação do Programa Mais Médicos. Dessa forma, fica evidente a necessidade do atendimento médico e humanizado para esse grupo social, utilizando os princípios do SUS, respeitando suas vontades, crenças e modos, tentando entendê-los de forma integral e dando todo o suporte que necessitam (SANDES LF, et al., 2018; MATOS PC, et al., 2018).

No entanto, é evidente que ainda existem obstáculos que impedem as melhorias das relações médicopaciente, como a falta de investimentos financeiros nos setores públicos, além da baixa qualidade na assistência dos profissionais. Assim, as melhorias feitas nesses âmbitos, partidas dos profissionais e dos pacientes, resultam na redução de danos que proporcionam um tratamento de sucesso (JASMIM JS e QUELUCI GC, 2018).

Ainda existem dificuldades relacionadas a alguns grupos sociais, como acesso ao serviço de saúde e obstáculo da comunicação com os profissionais de saúde, seja pela deficiência ou pelo idioma. Dessa forma, dentro do âmbito da saúde pública, percebe-se que existem algumas dificuldades encontradas por alguns grupos sociais, como acesso ao serviço, linguagem das cartilhas, além do obstáculo da comunicação com os profissionais de saúde. Percebe-se, assim a importância do exercício da comunicação e escuta reflexiva de fatores biopsicossociais do usuário contribui para uma atenção à saúde integrativa (SANTOS TD, et al., 2018; ARAÚJO AK, et al. 2015). Além disso, outro aspecto a ser analisado é a comunicação entre profissionais e usuários que utilizam os serviços públicos de saúde, quando esses apresentam diferentes idiomas. (MOURAO NETTO JJ, et al., 2018). 
Nesse aspecto, é de suma importância a aplicação de estratégias como linguagem não verbal, utilização de pausas durante a fala para a melhor compreensão, além da repetição e colaboração entre os profissionais que compõem o sistema são práticas fundamentais para a resolução de tais empecilhos (MOURAO NETTO JJ, et al., 2018; SAMUDIO JL, et al., 2017). É evidente, assim, que existem ferramentas cooperativas para a garantia do direito de acesso integral e universal de saúde a todos, baseados em estratégias que facilitam a qualidade da comunicação entre os que compõem o sistema de saúde (RANDOW R, et al., 2017; CHAVES SC, et al., 2019).

Assim, fica clara a necessidade de uma boa qualidade nas relações de saúde uma vez que, aos profissionais reconhecerem os usuários como um todo, considerando não apenas os aspectos biológicos, da doença, como também os seus valores e o fator biopsicossocial no qual ele se encontra inserido, para haver a garantia de uma maior eficácia nas condutas de comunicação para um melhor diagnóstico, tratamento e plano de cuidado desse usuário. Desse modo, observa-se a relevância da qualidade da comunicação e que seus efeitos afetam positivamente nas relações entre profissionais e usuários (SANDES LF, et al., 2018; CHAVES SC, et al., 2019).

\section{CONSIDERAÇÕES FINAIS}

Conclui-se que o Brasil é, de fato, um grande precursor nos avanços da saúde pública, principalmente, a partir do SUS, que garantiu o direito de acesso à saúde a todos, com atendimento integral e universal. No entanto, já no início da sua criação, foi possível observar fragilidades tanto no atendimento, como na falta de humanização na relação médico-paciente, o que gerava atendimentos genéricos e centrados apenas na doença, e não no paciente. Dessa forma, tornou-se necessária a implementação de políticas que suprisse essas lacunas. A Política Nacional de Humanização buscou estruturar tanto a prática clínica, o funcionamento, bem como a relação de comunicação entre gestores, trabalhadores e usuários SUS. Mas, mesmo com sua aplicabilidade, percebeu-se que é preciso ir além das fragilidades na comunicação verbal, do idioma ou do dialeto. Para garantir um atendimento humanizado, é preciso que o profissional se capacite, para que possa enxergar o paciente como um todo, biopsicossocial-político e inserido em um espaço, bem como haja investimentos financeiros nesta área, por parte dos gestores públicos.

\section{REFERÊNCIAS}

1. ARAUJO AK, et al. Dificuldades enfrentadas por enfermeiros na aplicabilidade da fitoterapia na atenção básica: uma revisão integrativa. Journal of Research Fundamental Care Online, 2015; 7(3):2826-2834

2. ASSIS WC, et al. Novas formas de cuidado através das práticas integrativas no sistema único de saúde. Revista Brasileira em Promoção da Saúde, 2017; 31(1):125-134.

3. BLUM RA, et al. O grupo "Trilhando Saúde": uma experiência para atuação na Atenção Básica à Saúde em Florianópolis. Revista Brasileira de Atividade Física \& Saúde, 2018; (23):e006.

4. CARVALHO JL, NÓBREGA MP. Práticas integrativas e complementares como recurso de saúde mental na Atenção Básica. Revista Gaúcha de Enfermagem, 2017; 38(4):e2017-0014.

5. CHAVES SC, et al. Intervenções não farmacológicas ofertadas ao usuário com transtorno mental comum na atenção primária à saúde. Journal Of Nursing And Health, 2019; 9(3):e199302.

6. CONTATORE OA, et al. Uso, cuidado e política das práticas integrativas e complementares na Atenção Primária à Saúde. Ciência e Saúde Coletiva, 2015; 20(10):3263-3273.

7. FERNANDES VC, et al. A participação comunitária no sistema único de saúde: revisão integrativa da literatura. Revista Brasileira em Promoção da Saúde, 2017; 30(1):125-134.

8. FUNDAÇÃO NACIONAL DE SAÚDE. Cronologia Histórica da Saúde Pública, 2017. Disponível em: http://www.funasa.gov.br/cronologia-historica-da-saude-publica. Acesso em: 17 abr. 2020.

9. GROSS DM, et al. Prevenção quaternária na gestão da atenção primária à saúde: revisão integrativa. Revista de enfermagem da UFPE online, 2016; (10):3608-3619.

10. JASMIM JS, QUELUCI GC. Estudos sobre pacientes diabéticos na atenção primária. Revista de enfermagem da UFPE online, 2018; 12(4):1072-1084.

11. MATOS PC, et al. Práticas Integrativas Complementares na Atenção Primária à Saúde. Cogitare Enfermagem, 2018; (23):e54781.

12. MATTA GC, PONTES AL. Políticas de Saúde: organização e operacionalização do sistema único de saúde. Rio de Janeiro: Fiocruz; 2018. Princípios e Diretrizes do Sistema Único de Saúde; p. 61-80.

13. MATTOS G, et al. Plantas medicinais e fitoterápicos na Atenção Primária em Saúde: percepção dos profissionais. Ciência e Saúde Coletiva, 2018; 23:3735-3744. 
14. MINISTÉRIO DA SAÚDE. Atenção Especializada; 2017. Disponível em: https://www.saude.gov.br/sistema-unico-desaude/estrutura-do-sus/770-sistema-nacional-de-saude/40317-atencao-especializada. Acesso em: 02 mai. 2020.

15. MINISTÉRIO DA SAÚDE. Humaniza SUS; 2017. Disponível em: https://www.saude.gov.br/acoes-eprogramas/humanizasus/sobre-o-programa. Acesso em: 02 mai. 2020.

16. MINISTÉRIO DA SAÚDE. Sistema Único de Saúde: estrutura, princípios e como funciona, 2019. Disponível em: https://www.saude.gov.br/sistema-unico-de-saude. Acesso em: 02 mai. 2020.

17. MOURÃO NETTO JJ, et al. Programa Mais Médicos e suas contribuições para a saúde no Brasil: revisão integrativa. Revista Panamericana de Salud Pública, 2018; 42(1):e2.

18. OLIVEIRA AF, et al. Fitoterapia na atenção básica: estudo com profissionais enfermeiros. Revista de Pesquisa: Cuidado é Fundamental Online, 2017; 8(2):480-487.

19. PEREIRA LB, CHAZAN AC. O Acesso das Pessoas Transexuais e Travestis à Atenção Primária à Saúde: uma revisão integrativa. Rev. Bras. de Med. Fam. Com., 2019; 14(41):1795-985.

20. RANDOW R, et al. Lian Gong em 18 terapias como estratégia de promoção da saúde. Revista Brasileira em Promoção da Saúde, 2017; 30(4):1-10.

21. SAMUDIO JL, et al. Agentes comunitários de saúde na atenção primária no brasil: multiplicidade de atividades e fragilização da formação. Trabalho, educação e saúde, 2017; 15(3):745-770.

22. SANDES LF, et al. Atenção primária à saúde de indígenas sul-americanos: revisão integrativa da literatura. Revista Panamericana de Salud Pública, 2018; 42:e163.

23. SANTOS TD, et al. Mutirão de saúde como ação extensiva e integrativa com a atenção primária. Revista Médica de Minas Gerais, 2018; 28:1-4.

24. SOARES DP, et al. Fatores Intervenientes na Realização das Práticas Integrativas e Complementares em Saúde na Atenção Básica pelos Enfermeiros. Revista de Enfermagem e Atenção à Saúde, 2019; 8(1):93-102.

25. SOUSA IM, TESSER CD. Medicina Tradicional e Complementar no Brasil: inserção no sistema único de saúde e integração com a atenção primária. Cadernos de Saúde Pública, 2017; 33(1):e00150215. 